\title{
The U Shaped Relationship Between High-Density Lipoprotein Cholesterol and All-Cause or Cause-Specific Mortality in Adult Population
}

This article was published in the following Dove Press journal: Clinical Interventions in Aging

\author{
Yu-qing Huang' \\ Xiao-cong Liu (D) \\ Kenneth Lo (iD ${ }^{1,2}$ \\ Lin Liu' \\ Yu-ling Yu $\mathbb{D}^{\prime}$ \\ Chao-lei Chen (D) \\ Jia-yi Huang' \\ Ying-qing Feng $\mathbb{D}^{\prime}$ \\ Bin Zhang'
}

'Department of Cardiology, Guangdong Cardiovascular Institute, Guangdong Provincial People's Hospital, Guangdong Academy of Medical Sciences, Guangzhou 510080, People's Republic of China:

${ }^{2}$ Department of Epidemiology, Centre for Global Cardio-Metabolic Health, Brown University, Providence, RI, USA
Correspondence: Ying-qing Feng; Bin Zhang

Department of Cardiology, Guangdong Cardiovascular Institute, Guangdong Provincial People's Hospital, Guangdong Academy of Medical Sciences, No. 106, Zhongshan Second Road, Yuexiu District, Guangzhou 510080, People's Republic of China

Tel/Fax +86-20-838278I 2

Email 651792209@qq.com;

3418989350@qq.com
Purpose: The associations of high-density lipoprotein cholesterol (HDL-C) with mortality are still unclear. We explored the associations of HDL-C with all-cause and cause-specific mortality in an adult population.

Methods: Deaths were classified into all-cause, cardiovascular, and cancer mortality. Survival curve, multivariate Cox regression, and subgroup analyses were conducted, and hazard ratio (HR) and 95\% confidence interval (CI) were performed. We fitted Cox regression models for all-cause, cardiovascular, and cancer mortality to evaluate their associations with categories of HDL-C ( $\leq 30,31-40,41-50,51-60$ [reference], 61-70, $>70 \mathrm{mg} / \mathrm{dL})$.

Results: A total of $42,145(20,415$ (48.44\%) males, mean age $47.12 \pm 19.40$ years) subjects were enrolled. At an average follow-up of $97.52 \pm 54.03$ months, all-cause, cardiovascular, and cancer mortality numbers were 5,061 (12.01), 1,081 (2.56\%), and 1,061 (2.52\%), respectively. When compared with the reference group (HDL-C: $51-60 \mathrm{mg} / \mathrm{dL}$ ), a U-shaped association was apparent for all-cause mortality, with elevated risk in participants with the lowest $(\leq 30 \mathrm{mg} / \mathrm{dL})(\mathrm{HR}=1.33 ; 95 \% \mathrm{CI}=1.14-1.56)$ and highest $(>70 \mathrm{mg} / \mathrm{dL})$ $(\mathrm{HR}=1.14 ; 95 \% \mathrm{CI}=1.02-1.27) \mathrm{HDL}-\mathrm{C}$ concentration. Associations for cardiovascular and cancer mortality were non-linear. An elevated risk for cancer mortality was observed in those with the highest HDL-C concentration ( $\mathrm{HR}=1.06$; 95\% CI-0.84-1.34) compared with the reference group, although it was not statistically significant. The effect of HDL-C on mortality was adjusted by some traditional risk factors including age, gender, race, or comorbidities.

Conclusion: A U-shaped association was observed between HDL-C and all-cause mortality among an adult population.

Keywords: high-density lipoprotein cholesterol, mortality, all-cause mortality, cause-specific mortality

\section{Introduction}

For the past several decades, it was generally believed that high-density lipoprotein cholesterol (HDL-C) was a blood lipid component that was beneficial to human health due to its protective role in the development of atherosclerosis. ${ }^{1}$ Numerous clinical and epidemiological studies also indicated that HDL-C played an important protective role in the occurrence of cardiovascular disease (CVD) or mortality across a wide range of concentrations. ${ }^{2-5}$ In the post hoc analysis of Treating to New Targets (TNT) study revealed that HDL-C levels were predictive of major cardiovascular events in patients treated with statins. ${ }^{6}$ However, recently some 
studies have suggested that HDL-C levels may not be predictive of CVD outcomes and has even paradoxically been associated with increased mortality in some subjects. The CANHEART study found that individuals with higher HDL-C levels had increased hazard of non-CVD mortality. ${ }^{7}$ A cohort study from China demonstrated that there was a U-shaped relationship between HDL-C levels and all-cause mortality, suggesting very high HDL-C levels may not represent a good prognosis. ${ }^{8}$ Considering the relationship between HDL-C and death is still unclear. Therefore, the aim of the present study was to evaluate the association of HDL-C levels with risk of all-cause and cause-specific (including cardiovascular and cancer) mortality in a large cohort study.

\section{Methods}

\section{Study Design and Population}

All the participants were the 1999-2014 National Health and Nutrition Examination Surveys (NHANES). The NHANES was a nationally representative survey of the civilian, non-institutionalized United States population conducted by the National Center for Health Statistics of the Center for Disease Control and Prevention. In this study, subjects with age $\geq 18$ years old with HDL-C measurement were enrolled. However, participants who were aged $<18$ years old, missing data on follow-up, missing HDL-C, and people with abnormal HDL-C (649 mg/dL) at baseline were excluded. After applying the exclusion criteria, a total of 42,145 participants were included for analysis (Figure 1). The survey protocol was approved by the Institutional Review Board of the Centers for Disease Control and Prevention. All participants have provided written informed consent.

\section{Data Collection}

The questionnaires and examinations in NHANES were performed on a standardized procedure and protocol. Baseline data mainly included socio-demographic information (such as age, gender, race, education level), lifestyle and behaviors (such as smoking status), comorbidities (such as hypertension, diabetes, CVD, and cancer), and current medication (such as hypoglycemic, antihypertensive and lipid-lowering drugs). Physical examination included height, weight, systolic blood pressure (SBP), and diastolic blood pressure (DBP). Body mass index (BMI) was defined as mass (kilograms) divided by the square of height (meters squared). Estimated glomerular filtration rate (eGFR) was calculated using Modification of Diet in Renal Disease formula. Hypertension was defined as having a history of hypertension, or SBP/DPB $\geq 140 / 90 \mathrm{mmHg}$, or using antihypertensive medications. ${ }^{9}$ Diabetes was defined as having a history of diabetes, or taking hypoglycemic medications currently, or fasting blood glucose level $\geq 7.0 \mathrm{mmol} / \mathrm{L} \mathrm{(126}$ $\mathrm{mg} / \mathrm{dL}$ ), or hemoglobin A1c (HbA1C) level $\geq 6.5 \%{ }^{10}$

\section{Lipids Measurement}

Sample collection and lipid measurement were based on a standardized protocol according to Centers for Disease Control and Prevention criteria. Blood samples were obtained from morning peripheral blood after fasting for at least 8 hours and shipped on dry ice to the laboratory analyzing the sample. Serum total cholesterol (TC) and triglycerides (TG) were measured enzymatically; HDL-C was measured by direct immunoassay or by precipitation. ${ }^{11}$ Serum HDL-C, TG, and TC levels were measured enzymatically at Johns Hopkins University Lipoprotein Analytic Laboratory with the use of a Hitachi 704 Analyzer

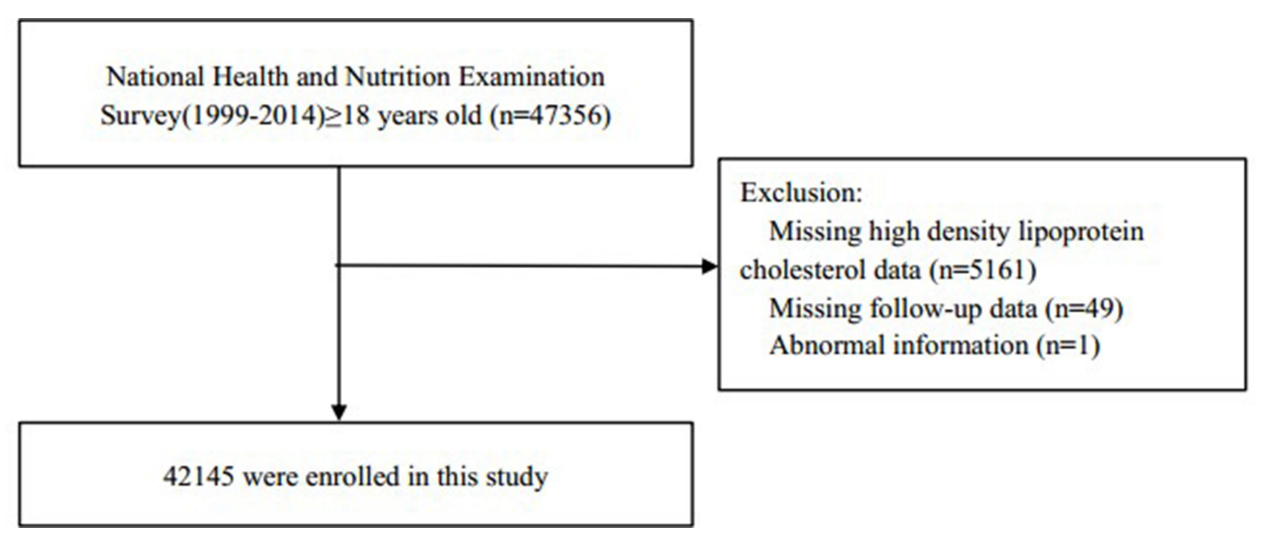

Figure I Research flow chart. 
(Boehringer Mannheim Diagnostics, Indianapolis, IN). ${ }^{12}$ Low density lipoprotein cholesterol (LDL-C) was derived with the use of the Friedewald formula [LDL-C $=$ TC $-\mathrm{HDL}-$ $\mathrm{C}-(\mathrm{TG} / 5)]$ if TG level was $\leq 400 \mathrm{mg} / \mathrm{dL}$. $^{13}$

\section{Outcome Assessment}

All-cause, cardiovascular, and cancer mortality were the outcomes of the present study. Mortality status was obtained from a publicly available dataset of the NHANES, which captured the vital status and cause of death of survey participants from baseline to December 31, 2015. For all-cause mortality, we included mortality from all causes. Cardiovascular mortality was defined by International Classification of Diseases, 10th Edition, Clinical Modification System codes (ICD-10) (I00-I09, I11, I13, and I20-I51) derived from death-certificate data. For cancer, mortality mainly included mortality from malignant neoplasms which were coded from $\mathrm{C} 00-\mathrm{C} 97$ in the ICD-10.

\section{Statistical Analyses}

Baseline continuous variables are presented as mean \pm standard deviation and categorical variables as a percentage where appropriate. Subgroup differences were analyzed by one-way ANOVA, Kruscal-Wallis $H$-test, chi-square, or Fisher tests. HDL-C were grouped into $\leq 30,31-40$, $41-50,51-60,61-70$, and $>70 \mathrm{mg} / \mathrm{dL}$, and the HDL-C concentration was $51-60 \mathrm{mg} / \mathrm{dL}$ as the reference group. Survival analysis was performed using standardized Kaplan-Meier curves and Log rank test. Multivariate Cox proportional hazards regression models were used to estimate hazard ratios (HRs) and 95\% confidence interval (CI) for all-cause, cardiovascular, and cancer mortality. Confounding variables including age, gender, race, education level, smoking, BMI, SBP, eGFR, energy intake, TC, comorbidities (hypertension, diabetes, CVD, and cancer), and medicine using (antihypertensive drugs, hypoglycemic agents, lipid-lowering drugs, and antiplatelet drugs). The shape of association between HDL-C levels and all-cause and cause-specific mortality was examined by multivariate adjusted Cox restricted cubic spline regression models, and used a generalized additive model to explore the nonlinear relationship between HDL-C and mortality. If a nonlinear relationship was detected, a two-piecewise Cox proportional hazards model on both sides of the inflection point, and log likelihood ratio test were performed. Subgroup analysis including age ( $<65$ or $\geq 65$ years), gender (male or female), race (White or non-White), BMI $\left(<25\right.$ or $\geq 25 \mathrm{~kg} / \mathrm{m}^{2}$ ), diabetes (yes or no), hypertension (yes or no), CVD (yes or no), and taking lipid-lowering drugs (yes or no), and analyzed their interactions between HDL-C levels with all-cause and cause-specific mortality. All statistical analyses were performed using $\mathrm{R}$ version 3.3.2 (R Foundation for Statistical Computing, Vienna, Austria), and $P<0.05$ was considered as statistically significant.

\section{Results}

\section{Baseline Characteristics}

The baseline characteristics of all the participants according to HDL-C levels are summarized in Table 1. A total of $42,145(20,415(48.44 \%)$ male) participants with an average age of $47.12 \pm 19.40$ years were enrolled. There were significant differences in age, gender, race, education level, smoking, BMI, SBP, DBP, TC, TG, LDL-C, eGFR, dietary energy, baseline CVD, diabetes, hypertension and cancer history, the use of statin, lipid-lowering, antihypertensive, antiplatelet and hypoglycemic drugs among groups according to HDL-C concentrations (all $P<0.05$ ).

\section{Incidence of Cause-Specific and All-Cause Mortality}

The incidence rate of all-cause and cause-specific mortality among HDL-C groups is shown in Table 1. During an average follow-up of $97.52 \pm 54.03$ months, there were $5,061(12.01 \%)$ cases of all-cause, $1,081(2.56 \%)$ cases of cardiovascular, and 1,061 (2.52\%) cases of cancer mortality. There were significant differences in all-cause, cardiovascular and cancer mortality among HDL-C groups. The cumulative survival probability of all-cause (Figure 2A), cardiovascular (Figure 2B), and cancer (Figure 2C) mortality among participants as stratified by HDL-C levels was demonstrated in Figure 2.

\section{HDL-C and All-Cause or Cause-Specific Mortality}

As shown in Figure 3, when compared with the reference group (HDL-C: $51-60 \mathrm{mg} / \mathrm{dL}$ ), after age, gender, race, education level, smoking, BMI, SBP, eGFR, energy intake, TC, comorbidities (hypertension, diabetes, CVD, and cancer), and medicine using (antihypertensive drugs, hypoglycemic agents, lipid-lowering drugs, and antiplatelet drugs) were adjusted, the multivariable adjusted HRs for all-cause mortality among HDL-C groups $(\leq 30,31-40,41-50$, $61-70,>70 \mathrm{mg} / \mathrm{dL})$ were $1.33(1.14-1.56), 1.15(1.04-$ 


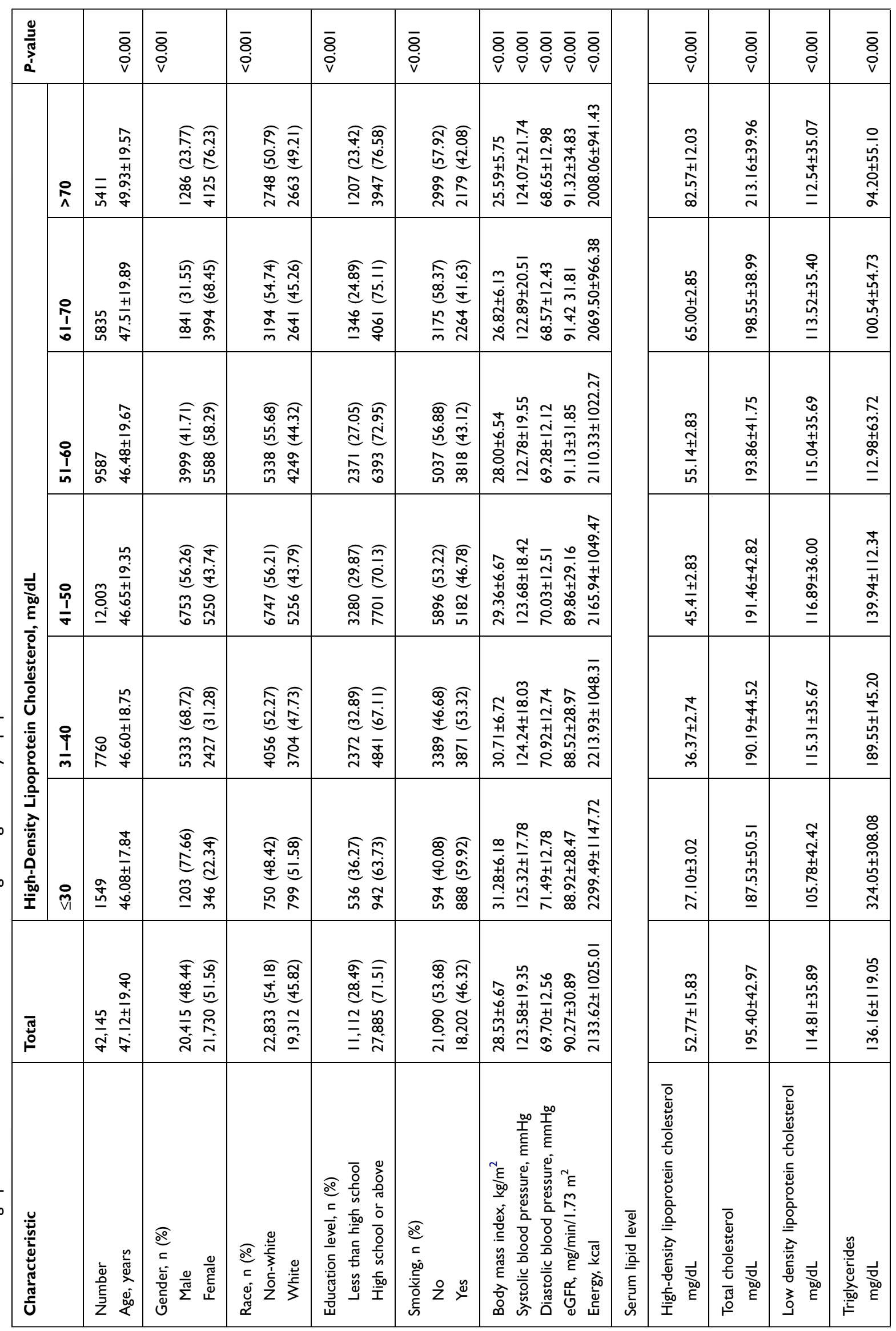




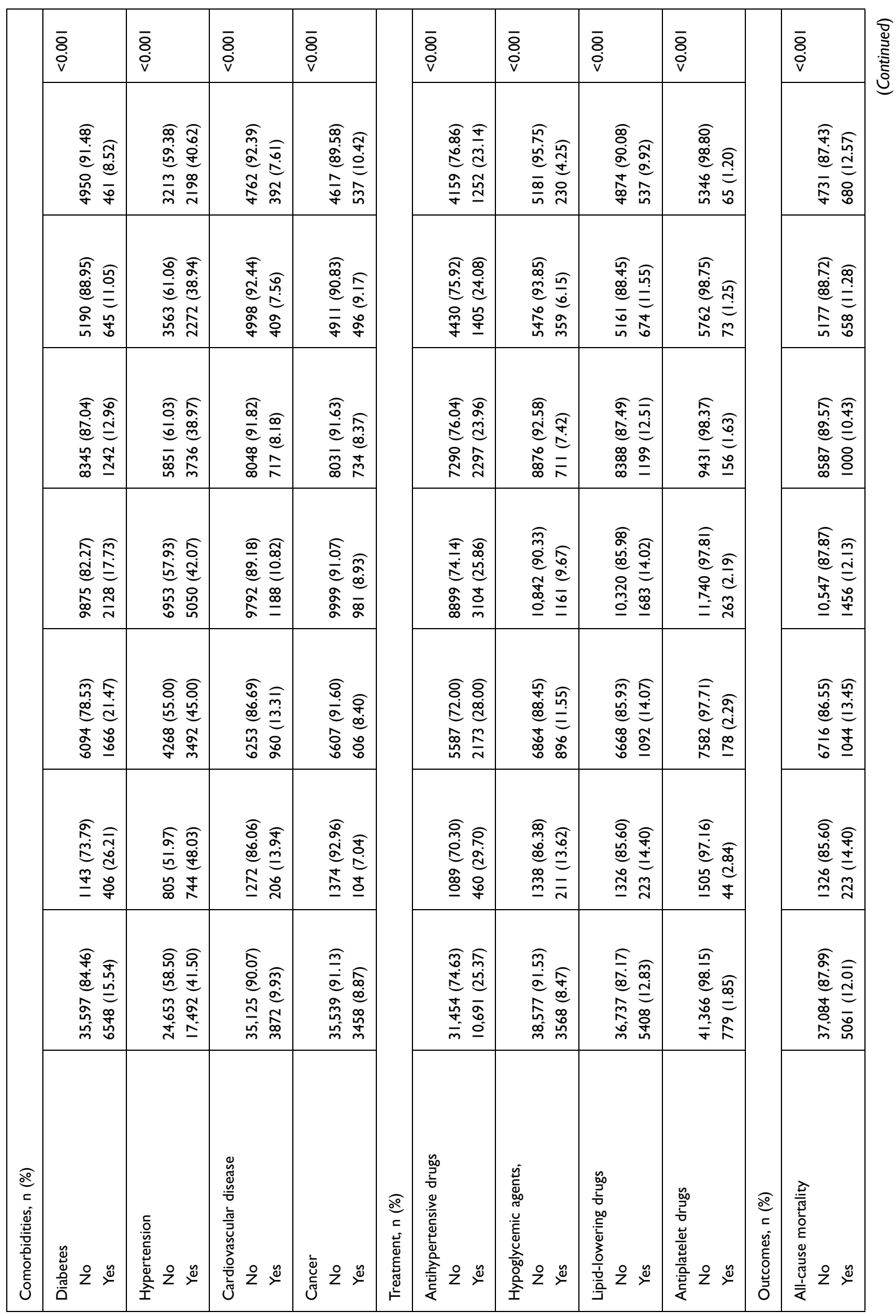




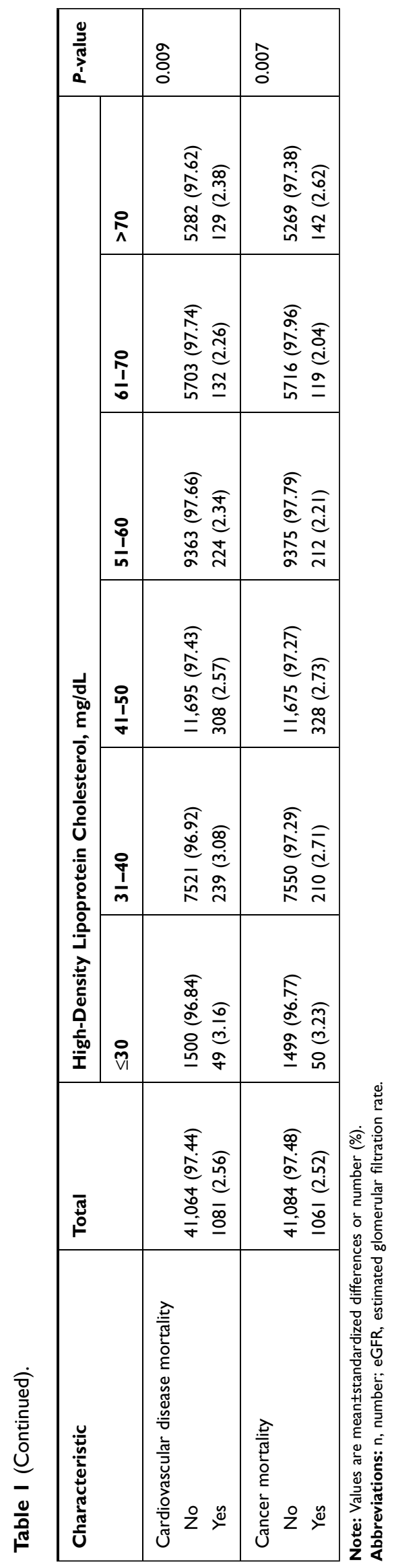

1.27), $1.11(1.02-1.22), 1.05(0.94-1.17)$ and $1.14(1.02-$ 1.27), the HRs for cardiovascular mortality were 1.24 (0.88-1.74), 1.09 (0.88-1.33), 0.96 (0.80-1.17), 0.92 (0.73-1.18) and $0.99(0.78-1.26)$, and the HRs for cancer mortality were 1.35 (0.97-1.88), 1.12 (0.91-1.38), 1.15 (0.96-1.38), 0.88 (0.69-1.12), and 1.06 (0.84-1.34), respectively.

The results of the two-piecewise linear regression model between HDL-C and mortality are demonstrated in Table 2, after adjusting for potential confounders, the cut-off values of all-cause, cardiovascular, and cancer mortality were $63 \mathrm{mg} / \mathrm{dL}, 46 \mathrm{mg} / \mathrm{dL}$, and $70 \mathrm{mg} / \mathrm{dL}$, respectively. On the left of cut-off value, the HRs for allcause, cardiovascular, and cancer mortality were 0.75 (95\% CI $=0.66-0.85 ; P<0.01), 0.51 \quad(95 \%$ CI $=0.29-0.88$; $P=0.01)$, and $0.67(95 \% \mathrm{CI}=0.52-0.85 ; P<0.01)$ for every $1 \mathrm{mmol} / \mathrm{L}(38.66 \mathrm{mg} / \mathrm{dL})$ raise in HDL-C, while on the right of the cut-off value, the HRs for all-cause and cardiovascular mortality were $1.51 \quad(95 \% \quad \mathrm{CI}=1.30-1.76$; $P<0.01), 1.11 \quad(95 \% \quad \mathrm{CI}=0.89-1.40 ; \quad P=0.36)$, and 1.7 $(95 \% \mathrm{CI}=1.20-2.53 ; P<0.01)$, respectively. After adjusting for various potential confounders, as shown in Figure 4, the association between HDL-C on a continuous scale and all-cause, cardiovascular and cancer mortality appear to be U-shaped, as both low and high concentrations were associated with high all-cause (Figure 4A), cardiovascular (Figure 4B) and cancer (Figure 4C) mortality.

\section{Subgroup Analyses}

As shown in Table 3, after multivariate adjustment for confounders, when performing subgroup analysis using age, gender, race, BMI, diabetes, hypertension, CVD, and taking lipid-lowering drugs, we found that there was a U-shaped relationship between HDL-C and all-cause mortality in the male population, subjects aged $\geq 65$ years, the non-White population, and hypertension patients. Similarly, this study also showed that, in male subjects, HDL-C showed a U-shaped relationship with cancer mortality. Gender, race, BMI, and hypertension interacted significantly with the association between HDL-C levels and all-cause mortality (both $P$ for interaction $<0.05$ ), while only race interacted significantly with HDL-C levels to influence the association with cardiovascular mortality $(P$-interaction $<0.01)$. In addition, the relationship between HDL-C and cancer mortality was affected by age and baseline CVD history (both $P$ for interaction $<0.05$ ). 


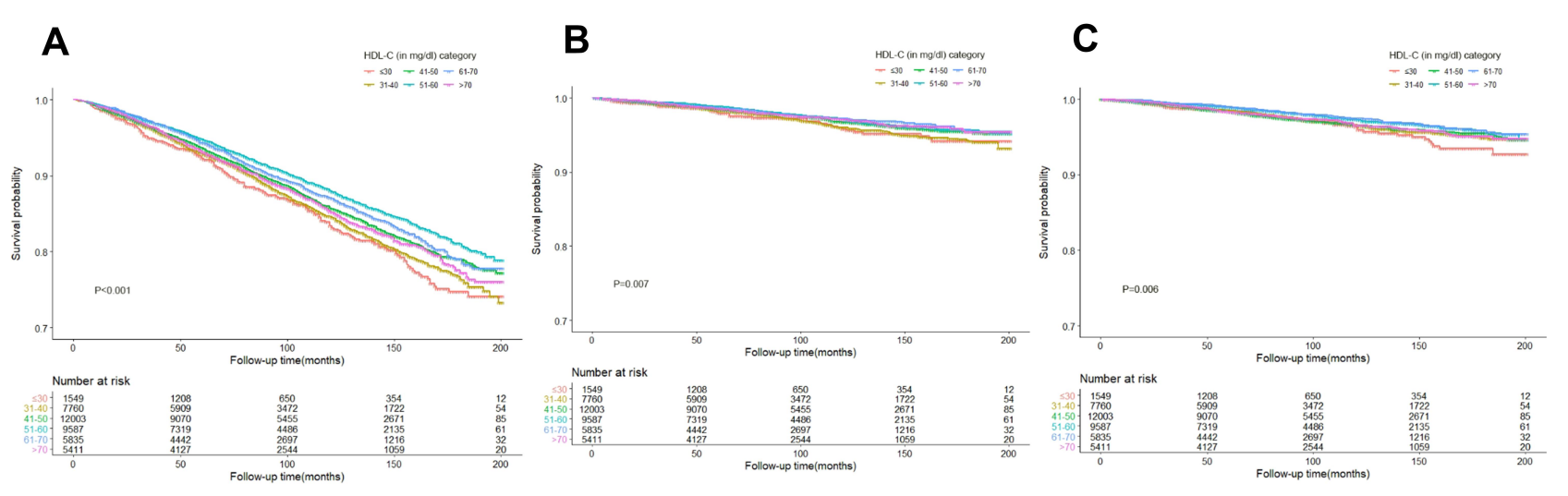

Figure 2 Kaplan-Meier survival curves for all-cause (A), cardiovascular (B), and cancer (C) mortality.

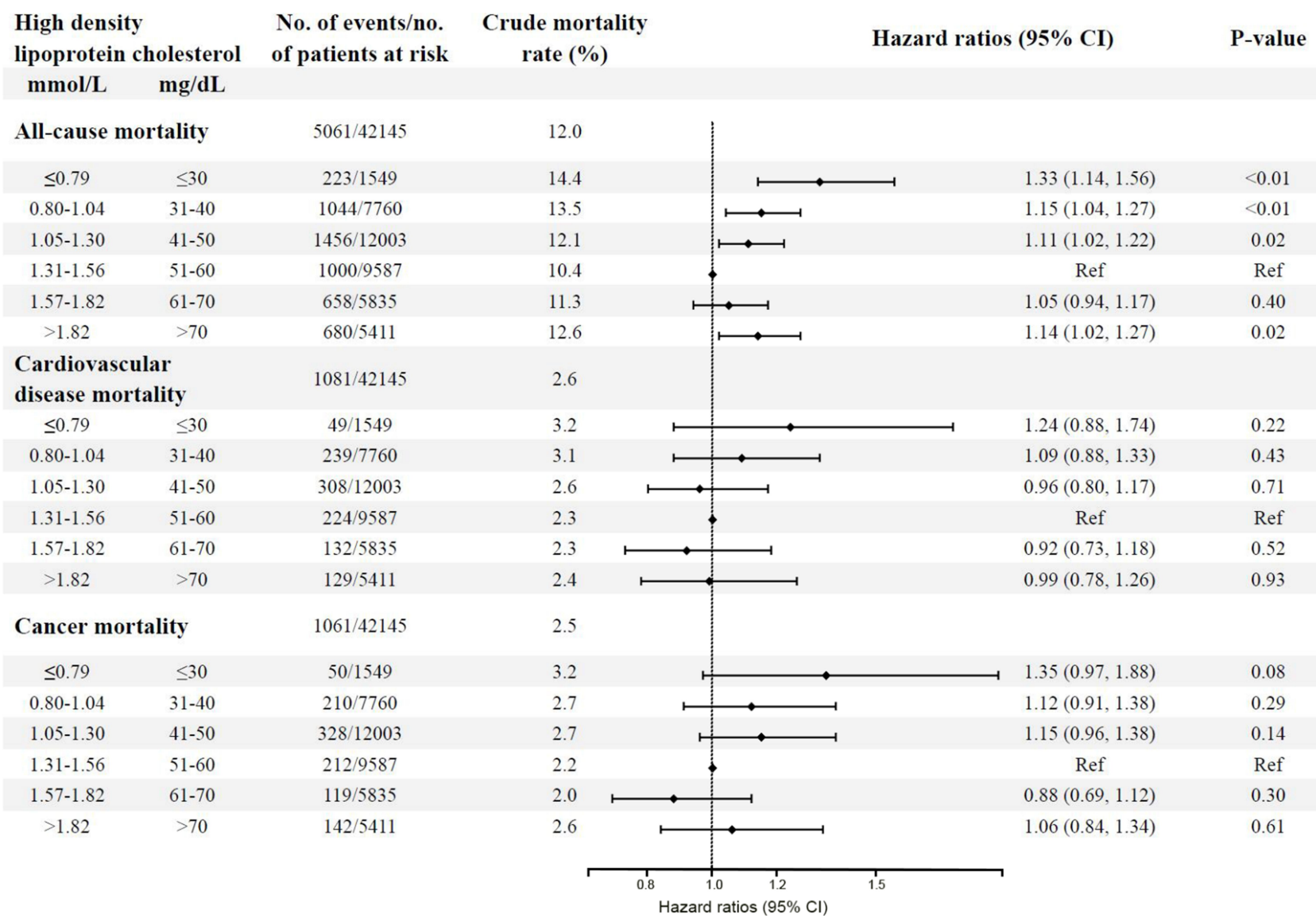

Figure 3 The relationship between high-density lipoprotein cholesterol and mortality. Age, gender, race, education level, smoking, body mass index, systolic blood pressure, estimated glomerular filtration rate, energy intake, total cholesterol, comorbidities (hypertension, diabetes, cardiovascular disease, and cancer), and medicine using (antihypertensive drugs, hypoglycemic agents, lipid-lowering drugs, and antiplatelet drugs) were adjusted.

Abbreviations: $\mathrm{Cl}$, confidence interval; Ref, reference.

\section{Discussion}

In this large cohort of general adults population we found that HDL-C has a detailed U-shaped relationship with all-cause mortality, but has a non-linear relationship with cardiovascular and cancer mortality. Higher or lower HDL-C levels may increase the risk of all- cause and specific deaths. There was an interaction between gender, race, BMI, hypertension, and allcause mortality, while only race has a significant interaction with cardiovascular mortality, and age and baseline CVD history has a significant interaction with cancer mortality. 
Table 2 The Results of Two-Piecewise Linear Regression Model Between High-Density Lipoprotein Cholesterol and Mortality

\begin{tabular}{|c|c|c|c|}
\hline & $\begin{array}{l}\text { All-Cause Mortality } \\
\text { HR (95\% CI) P-value }\end{array}$ & $\begin{array}{l}\text { Cardiovascular Disease Mortality } \\
\text { HR }(95 \% \mathrm{Cl}) \text { P-value }\end{array}$ & $\begin{array}{l}\text { Cancer Mortality } \\
\text { HR ( } 95 \% \mathrm{CI}) \text { P-value }\end{array}$ \\
\hline Cutoff value, $\mathrm{mg} / \mathrm{dL}$ & 63 & 46 & 70 \\
\hline$<$ Cut-off value & $0.75(0.66-0.85)<0.01$ & $0.51(0.29-0.88) 0.01$ & $0.67(0.52-0.85)<0.01$ \\
\hline$\geq$ Cut-off value & $1.51(1.30-1.76)<0.01$ & I.II (0.89-I.40) 0.36 & $1.74(1.20-2.53)<0.01$ \\
\hline$P$ for log likelihood ratio test & $<0.01$ & 0.02 & $<0.01$ \\
\hline
\end{tabular}

Notes: Age, gender, race, education level, smoking, body mass index, systolic blood pressure, estimated glomerular filtration rate, energy, total cholesterol, comorbidities (hypertension, diabetes, cardiovascular disease, and cancer), and medicine use (antihypertensive drugs, hypoglycemic agents, lipid-lowering drugs, and antiplatelet drugs) were adjusted.

Abbreviations: $\mathrm{HR}$, hazard ratio; $\mathrm{Cl}$, confidence interval.

Our results were consistent with previous studies, ${ }^{8,-14-16}$ indicating that a U-shaped association was observed between HDL-C and all-cause mortality. In addition, we also showed that HDL-C has a nonlinear relationship with cardiovascular and tumor death, rather than the typical U-shaped relationship. That was, low-level HDL-C has a higher risk of cause-specific mortality, but higher HDL-C; although it also has a higher risk of mortality, this was not statistically significant in cardiovascular mortality. Joseph et $\mathrm{al}^{17}$ found that low HDL-C was associated with risk of higher cardiovascular and malignancy mortality, but high HDL-C was associated with lower risk of cardiovascular and malignancy mortality. In addition, a study from an elderly population demonstrated that cardiovascular mortality was significantly highest in the lowest quartile of HDL$\mathrm{C}$, and particularly low levels of HDL-C seem to be risk factors for cardiovascular mortality. ${ }^{18}$ There was also a study which found that individuals with lower HDL-C levels were independently associated with higher risk of CVD and cancer mortality compared with individuals in the reference ranges of HDL-C levels, and individuals with higher HDL levels had increased hazard of non-CVD mortality. ${ }^{7}$ The reason our study was different from previous studies may be mainly due to the different populations. In addition, the adjustment of different confounding factors may also have a certain effect on the results.

We also found that the relationship between HDL-C and all-cause, cardiovascular, and cancer mortality were all non-linear, and the cut-off values were different. When HDL-C was less than $63 \mathrm{mg} / \mathrm{dL}, 46 \mathrm{mg} / \mathrm{dL}$, and $70 \mathrm{mg} / \mathrm{dL}$, respectively, the lower the HDL-C, the higher the allcause, cardiovascular, and cancer death risk. However, when HDL-C was greater than the above cut-off values, higher HDL-C was accompanied by a higher all-cause, cardiovascular, and cancer death risk, but there was no statistical significance in cardiovascular death. Previous study has shown that in both gender, HDL-C 31-40 mg/ $\mathrm{dL}$ and $\leq 30 \mathrm{mg} / \mathrm{dL}$ were associated with higher risk of allcause mortality, cardiovascular mortality, malignancyrelated deaths, and HDL-C $>60 \mathrm{mg} / \mathrm{dL}$ was associated with lower all-cause, cardiovascular, malignancy-related deaths. ${ }^{17}$ Sun et $\mathrm{al}^{8}$ found that very high HDL-C levels ( $\geq 80 \mathrm{mg} / \mathrm{dL}$ ) were independently associated with increased total mortality risk compared with the reference level. In

A

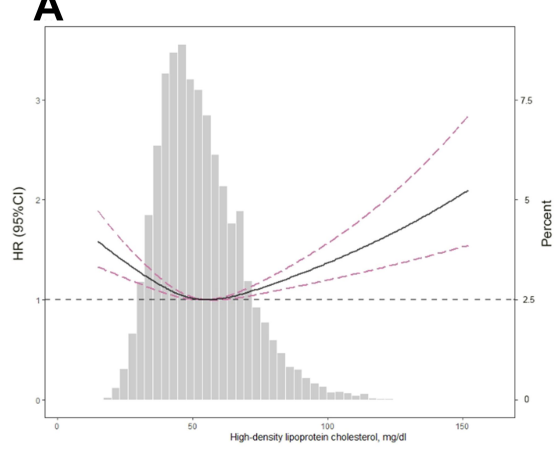

B

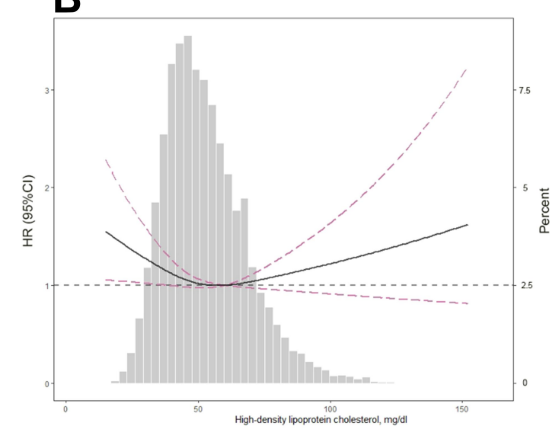

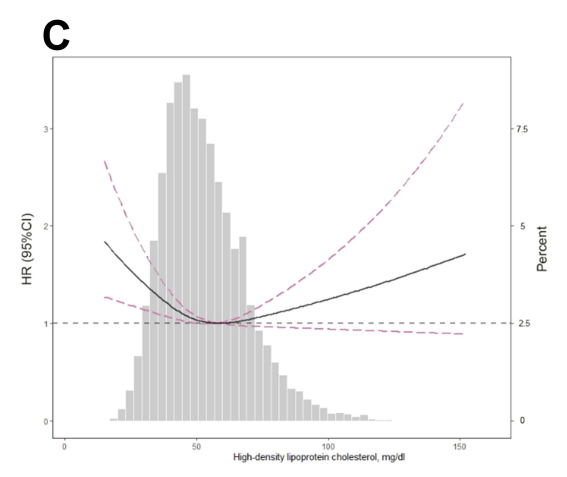

Figure 4 Adjusted spline curves analyze for all-cause (A), cardiovascular (B), and cancer (C) mortality by high-density lipoprotein cholesterol (HDL-C) levels in the overall cohort and the HDL-C probability distribution histogram is represented in the background. Age, gender, race, education level, smoking, body mass index, systolic blood pressure, estimated glomerular filtration rate, energy intake, total cholesterol, comorbidities (hypertension, diabetes, cardiovascular disease, and cancer), and medicine using (antihypertensive drugs, hypoglycemic agents, lipid-lowering drugs, and antiplatelet drugs) were adjusted. 


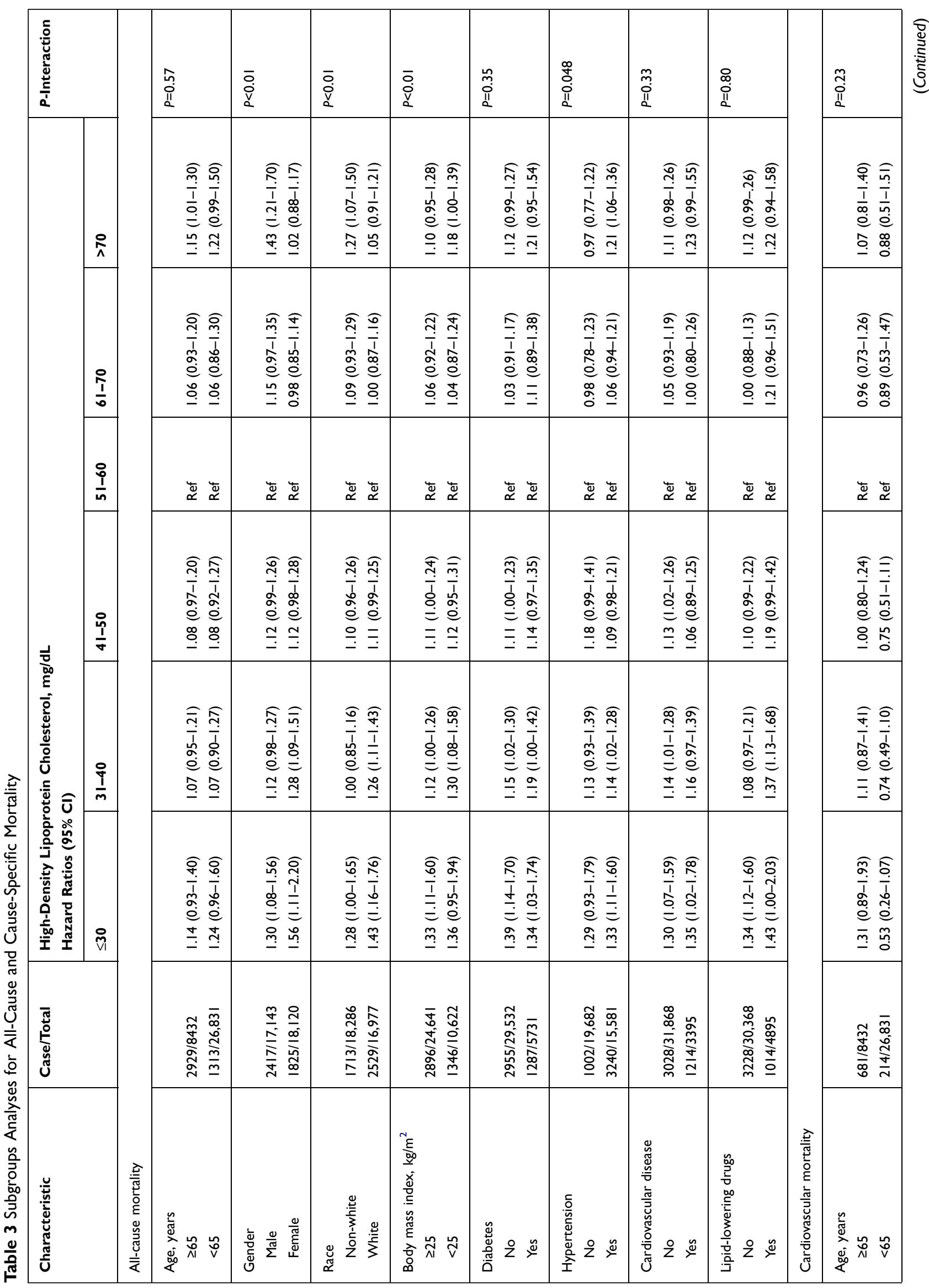




\begin{tabular}{|c|c|c|c|c|c|c|c|c|c|c|c|}
\hline 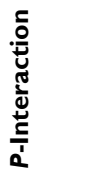 & & $\frac{t}{i}$ & $\begin{array}{l}\bar{\partial} \\
\dot{v} \\
\Sigma\end{array}$ & $\begin{array}{l}\infty \\
0 \\
\text { II } \\
0\end{array}$ & $\begin{array}{l}\text { D } \\
\text { iा } \\
\text { II }\end{array}$ & $\begin{array}{l}\hat{n} \\
\hat{i} \\
\text { in }\end{array}$ & $\begin{array}{l}\text { ồ } \\
\text { II } \\
\text { in }\end{array}$ & $\frac{0}{i}$ & & $\begin{array}{l}\bar{\partial} \\
\dot{v} \\
\Sigma\end{array}$ & $\frac{m}{i}$ \\
\hline & 웃 & 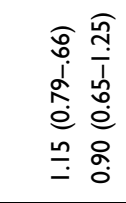 & 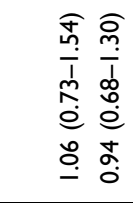 & 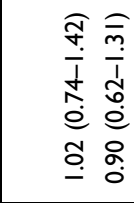 & 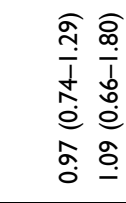 & 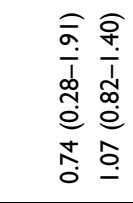 & 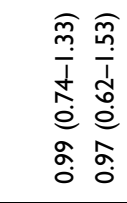 & 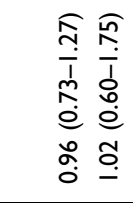 & & 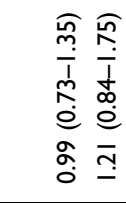 & 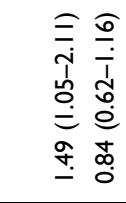 \\
\hline & $\frac{0}{\frac{1}{6}}$ & 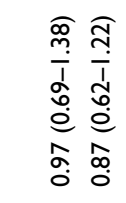 & 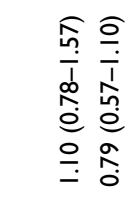 & 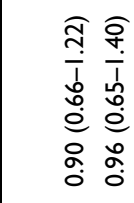 & 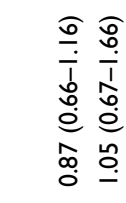 & 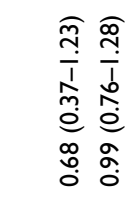 & 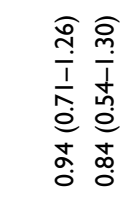 & 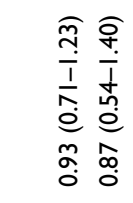 & & 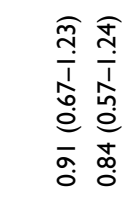 & 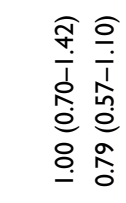 \\
\hline & $\frac{i}{i}$ & $\begin{array}{l}\overleftarrow{\Phi} \\
\check{\Phi}\end{array}$ & $\begin{array}{ll}\overleftarrow{\Phi} \\
\check{\Phi}\end{array}$ & 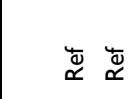 & $\begin{array}{ll}\overleftarrow{\Phi} & \overleftarrow{\Phi} \\
\propto & \end{array}$ & 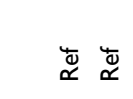 & 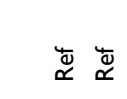 & 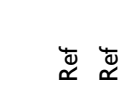 & & ¿্ষ & ¿্ষ \\
\hline & $\begin{array}{l}\frac{0}{p} \\
\frac{1}{\sigma}\end{array}$ & 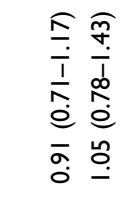 & 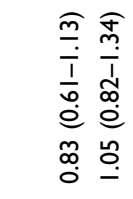 & 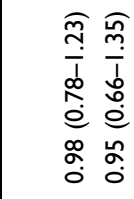 & 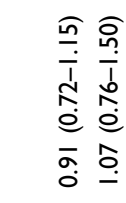 & 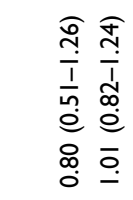 & 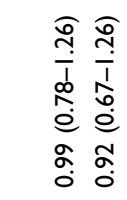 & 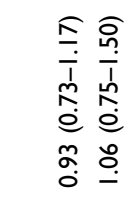 & & 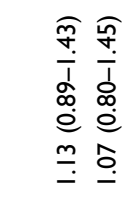 & 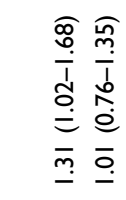 \\
\hline 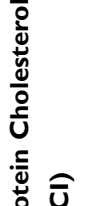 & $\frac{q}{m}$ & 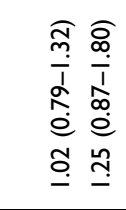 & 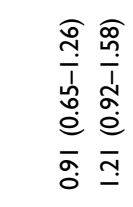 & 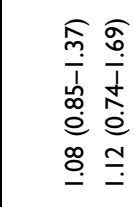 & 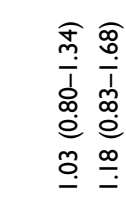 & 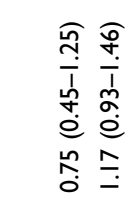 & 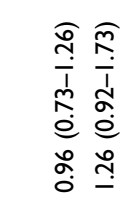 & 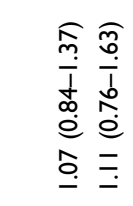 & & 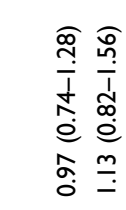 & 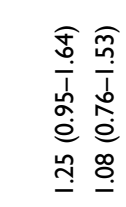 \\
\hline 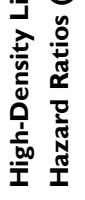 & 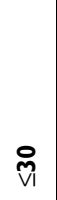 & 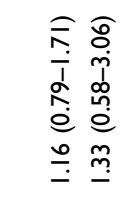 & 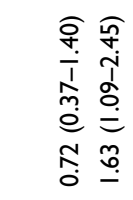 & 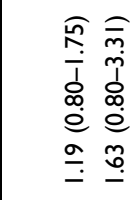 & 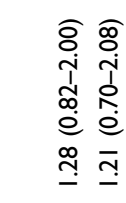 & 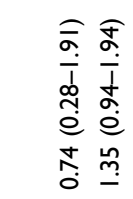 & 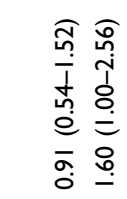 & 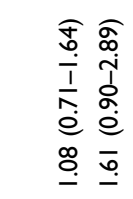 & & 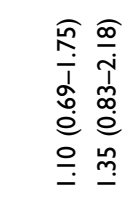 & 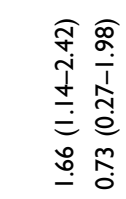 \\
\hline סٌ & & 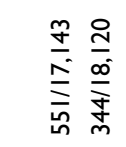 & 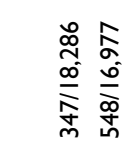 & 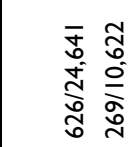 & 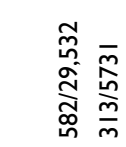 & 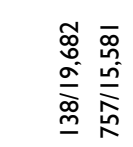 & 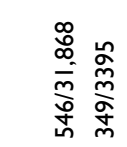 & 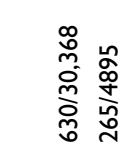 & & 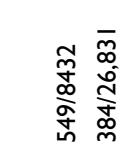 & 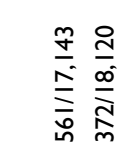 \\
\hline 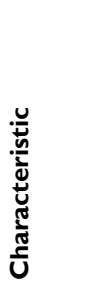 & & 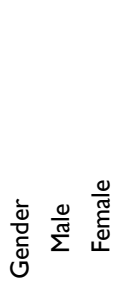 & 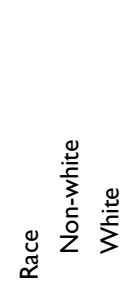 & 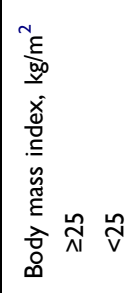 & 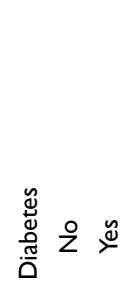 & 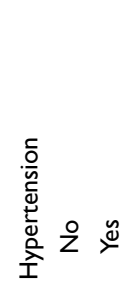 & 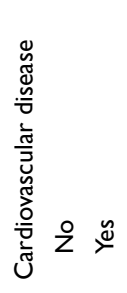 & 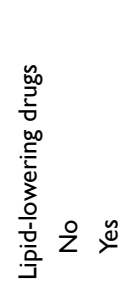 & 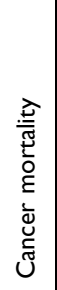 & 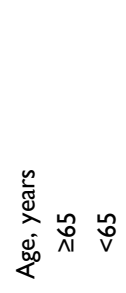 & 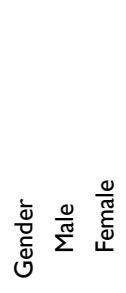 \\
\hline
\end{tabular}




\begin{tabular}{|c|c|c|c|c|c|}
\hline $\begin{array}{l}\text { 옹 } \\
\text { III }\end{array}$ & $\begin{array}{l}\text { นn } \\
\text { II } \\
\text { in }\end{array}$ & 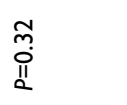 & $\frac{1}{i}$ & $\begin{array}{l}\bar{\partial} \\
\dot{v} \\
\Sigma\end{array}$ & $\begin{array}{l}\text { fे } \\
\text { II } \\
\text { in }\end{array}$ \\
\hline 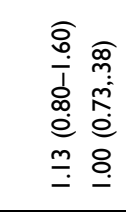 & 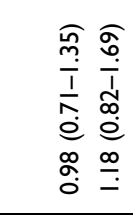 & 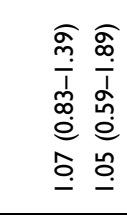 & 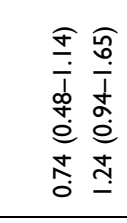 & 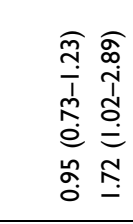 & 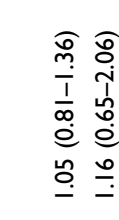 \\
\hline 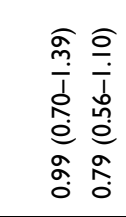 & 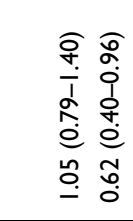 & 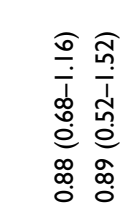 & 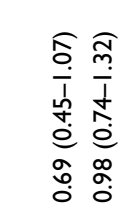 & 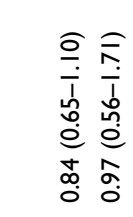 & 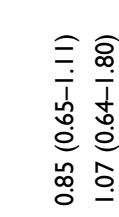 \\
\hline 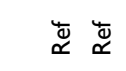 & $\stackrel{\bar{\Xi}}{\check{\Phi}}$ & $\begin{array}{l}\overleftarrow{\Phi} \\
\check{\Phi}\end{array}$ & $\begin{array}{l}\grave{\Phi} \\
\check{\Phi}\end{array}$ & $\begin{array}{l}\overleftarrow{\Phi} \\
\mathscr{\Phi}\end{array}$ & 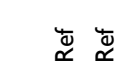 \\
\hline 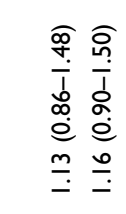 & 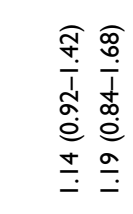 & 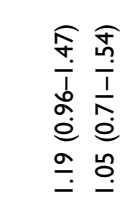 & 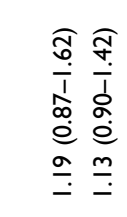 & 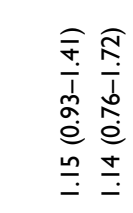 & 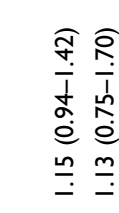 \\
\hline 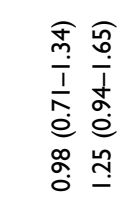 & 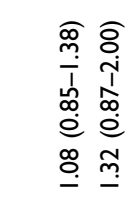 & 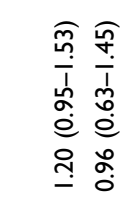 & 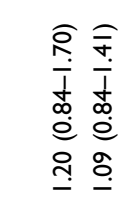 & 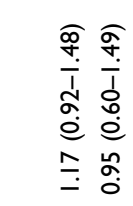 & 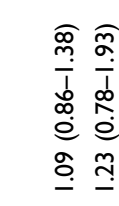 \\
\hline 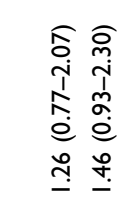 & 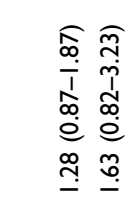 & 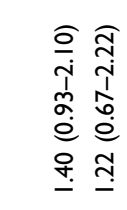 & 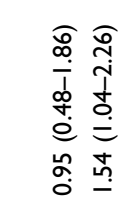 & 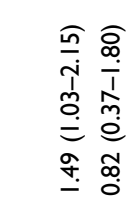 & 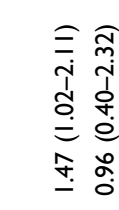 \\
\hline 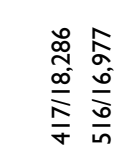 & 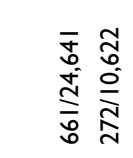 & 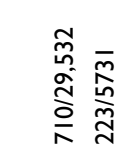 & 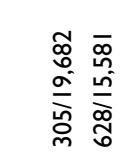 & 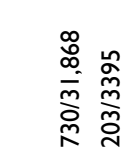 & 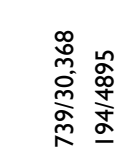 \\
\hline 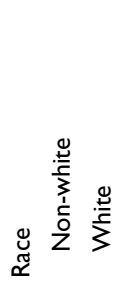 & 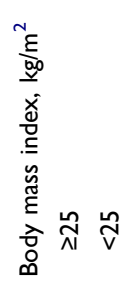 & 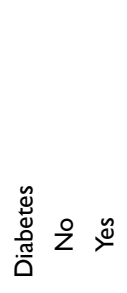 & 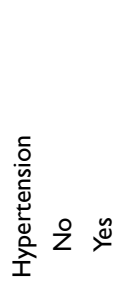 & 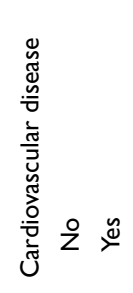 & 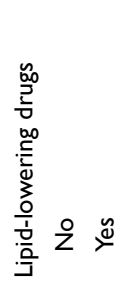 \\
\hline
\end{tabular}


addition, a cohort study from South Korea showed that participants with HDL-C level $\geq 85 \mathrm{mg} / \mathrm{dL}$ had the lowest HRs for cardiovascular deaths, participants with HDL-C level of $62-69 \mathrm{mg} / \mathrm{dL}$ showed the lowest proportion of overall deaths and cancer deaths. ${ }^{19}$ It also indicated that a very high HDL-C level ( $\geq 85 \mathrm{mg} / \mathrm{dL}$ ) was associated with increased risk of all-cause deaths. ${ }^{19}$ The prospective cohort study from Japanese adults showed that very high HDL-C ( $\geq 80 \mathrm{mg} / \mathrm{dL})$ did not show a significant association with CVD and other cause-specific mortality. ${ }^{20}$ In summary, very high HDL-C may also increase the risk of allcause or cause-specific deaths.

A subgroup analysis of our study demonstrated that the relationship between HDL-C and all-cause or cause-specific mortality was different in gender, age, race, taking lipid-lowering drugs, and this relationship was adjusted and interacted by some traditional risks, such as age, gender, and comorbidities. Li et $\mathrm{al}^{21}$ found that there were nonlinear associations of HDL-C with all-cause and cardiovascular mortality among the elderly population, and the optimal HDL-C level and range were $71 \mathrm{mg} / \mathrm{dL}$ and 61 to $87 \mathrm{mg} / \mathrm{dL}$, respectively. The REGARDS study showed that low HDL-C was associated with reduced risk of incident CVD in black participants, and very low HDL$\mathrm{C}$ in women was significantly associated with cancer mortality in a fully adjusted model. ${ }^{2}$ A cohort study from American adults found that both extremely low and high HDL-C levels were associated with greater risk of mortalities, and Mexican-American ethnicity subjects in the low level of HDL-C (30-40 mg/dL) category had higher risk of mortalities than those with a very low level, suggesting an HDL-C paradox in Mexican-American ethnicity participants. ${ }^{22}$ Moreover, a non-dialysis-dependent chronic kidney disease population revealed that HDL-C $>60 \mathrm{mg} /$ $\mathrm{dL}$ was associated with lower risk of all-cause, cardiovascular, and malignant mortality in women but not in men. ${ }^{17}$

At present, the paradox mechanism of HDL-C and mortality is still unclear. The main reason may be as follows; on the one hand, the increase in adverse events observed in some trials where HDL-C was raised in large amounts could be related with some other CVD risks more than the HDL-C increase itself. On the other hand, the association between extreme high HDL-C and higher mortality was that extremely high HDL-C concentrations may be due to genetic variation of certain genes, such as CETP, ABCA1, LIPC, and SCARB $1 .{ }^{23}$ In addition, the exact mechanism of higher HDL-C levels with higher risk of mortality may be mainly due to the function of HDL. It has been demonstrated that measurement of HDL functionality indices, independent of HDL-C assessment, was a more robust tool for the evaluation of the functional status of HDL and CVD risks. ${ }^{24}$ Consistent with HDL complexity in composition and metabolism, a wide range of biological activities was reported for HDL, including antioxidant, anti-inflammatory, anti-thrombotic, and immune modulatory activities. ${ }^{25}$ Finally, HDL-C included different particle size and number, in which the shape, electrophoresis speed, lipid and protein composition were also different, and the predictive ability of cardiovascular events was also inconsistent. Therefore, recent researches were mainly focused on improving HDL functionality, rather than paying too much attention to HDL-C levels.

The current study has several strengths. First, the enrolled population was obtained from a nationally representative survey. Second, the follow-up time of this study was relatively long and there were large number of samples and events. Finally, the NHANES adopted standard procedures and methods to data collection, and used strict methods for data quality control. Despite these strengths, there were several limitations in this study. First, some baseline variables such as previous disease history and history of taking medication were self-reported, which may be some recall errors. Second, other covariates, such as inflammation marker, physical activity, and uric acid, which may also have an effect on cause-specific and all-cause mortality. Third, blood lipids were only measured once at baseline, which may not truly reflect the participant's blood lipid status. Finally, our research only focused on HDL-C, without analyzing other blood lipids, or other potentially important aspects of HDL$\mathrm{C}$, such as particle sizes and subclasses of HDL-C.

In conclusion, in the present study, a U-shaped association was observed between HDL-C and all-cause mortality among an adult general population, and this association was modified by gender, race, BMI, and hypertension. In addition, as for cause-specific (including cardiovascular and cancer mortality) there was a nonlinear relations ion HDL-C and them. When HDL-C levels were greater than $63 \mathrm{mg} / \mathrm{dL}, 46$ $\mathrm{mg} / \mathrm{dL}$, and $70 \mathrm{mg} / \mathrm{dL}$, it may increase the risk of all-cause, cardiovascular, and cancer mortality, respectively. The relationship between HDL-C and cause-specific deaths is still unclear. In the future more well-designed prospective cohort studies are needed to clarify the association of HDL-C and all-cause or cause-specific mortality.

\section{Author Contributions}

All authors made substantial contributions to the conception and design, acquisition of data, or analysis and 
interpretation of data; took part in drafting the article or revising it critically for important intellectual content; agreed on the journal to which the article will be submitted; gave final approval of the version to be published; and agree to be accountable for all aspects of the work.

\section{Funding}

This research was supported by Science and Technology Plan Program of Guangzhou (No.201604020143, No.201604 020018, No.201604020186 and No.201803040012), the National Key Research and Development Program of China (No.2017YFC1307603 and No.2016YFC1301305) and the Key Area R\&D Program of Guangdong Province (No.2019B020227005).

\section{Disclosure}

The authors of this paper reported no financial conflicts of interest.

\section{References}

1. Otocka-Kmiecik A, Mikhailidis DP, Nicholls SJ, et al. Dysfunctional HDL: a novel important diagnostic and therapeutic target in cardiovascular disease? Prog Lipid Res. 2012;51(4):314-324. doi:10.1016/j. plipres.2012.03.003

2. Penson P, Long DL, Howard G, et al. Associations between cardiovascular disease, cancer, and very low high-density lipoprotein cholesterol in the REasons for Geographical and Racial Differences in Stroke (REGARDS) study. Cardiovasc Res. 2019;115(1):204-212. doi:10.1093/cvr/cvy198

3. Silbernagel G, Schottker B, Appelbaum S, et al. High-density lipoprotein cholesterol, coronary artery disease, and cardiovascular mortality. Eur Heart J. 2013;34(46):3563-3571. doi:10.1093/eurheartj/eht343

4. Gordon T, Castelli WP, Hjortland MC, Kannel WB, Dawber TR. High density lipoprotein as a protective factor against coronary heart disease. The Framingham Study. Am J Med. 1977;62(5):707-714. doi:10.1016/0002-9343(77)90874-9

5. Laks R, Araujo LM, Almada FCM, Cendoroglo MS. The importance of HDL-C and CRP in cardiovascular risk evaluation in longevous elderly individuals. Einstein (Sao Paulo). 2011;9(3):397-403. doi:10.1590/s1679-45082011rw1953

6. Barter P, Gotto AM, LaRosa JC, et al. HDL cholesterol, very low levels of LDL cholesterol, and cardiovascular events. $N$ Engl J Med. 2007;357(13):1301-1310. doi:10.1056/NEJMoa064278

7. Ko DT, Alter DA, Guo H, et al. High-density lipoprotein cholesterol and cause-specific mortality in individuals without previous cardiovascular conditions: the CANHEART study. J Am Coll Cardiol. 2016;68 (19):2073-2083. doi:10.1016/j.jacc.2016.08.038

8. Li X, Guan B, Wang Y, et al. Association between high-density lipoprotein cholesterol and all-cause mortality in the general population of northern China. Sci Rep. 2019;9(1):14426. doi:10.1038/s41598019-50924-4

9. James PA, Oparil S, Carter BL, et al. 2014 evidence-based guideline for the management of high blood pressure in adults: report from the panel members appointed to the Eighth Joint National Committee (JNC 8). JAMA. 2014;311(5):507-520. doi:10.1001/ jama.2013.284427
10. Association AD. 2. Classification and diagnosis of diabetes: standards of medical care in diabetes-2018. Diabetes Care. 2018;41(Suppl 1): S13-S27. doi: $10.2337 / \mathrm{dc} 18-\mathrm{S} 002$

11. Bucholz EM, Rodday AM, Kolor K, Khoury MJ, de Ferranti SD. Prevalence and predictors of cholesterol screening, awareness, and statin treatment among US adults with familial hypercholesterolemia or other forms of severe dyslipidemia (1999-2014). Circulation. 2018;137(21):2218-2230. doi:10.1161/CIRCULATIONAHA.117.03 2321

12. Doran B, Guo Y, Xu J, et al. Prognostic value of fasting versus nonfasting low-density lipoprotein cholesterol levels on long-term mortality: insight from the National Health and Nutrition Examination Survey III (NHANES-III). Circulation. 2014;130 (7):546-553. doi:10.1161/CIRCULATIONAHA.114.010001

13. Friedewald WT, Levy RI, Fredrickson DS. Estimation of the concentration of low-density lipoprotein cholesterol in plasma, without use of the preparative ultracentrifuge. Clin Chem. 1972;18(6):499-502. doi:10.1093/clinchem/18.6.499

14. Hamer M, O'Donovan G, Stamatakis E. High-density lipoprotein cholesterol and mortality: too much of a good thing? Arterioscler Thromb Vasc Biol. 2018;38(3):669-672. doi:10.1161/ATVBAHA. 117.310587

15. Bowe B, Xie Y, Xian H, et al. High density lipoprotein cholesterol and the risk of all-cause mortality among U.S. veterans. Clin J Am Soc Nephrol. 2016;11(10):1784-1793. doi:10.2215/CJN.00730116

16. DeGoma EM, Leeper NJ, Heidenreich PA. Clinical significance of high-density lipoprotein cholesterol in patients with low low-density lipoprotein cholesterol. J Am Coll Cardiol. 2008;51(1):49-55. doi:10.1016/j.jacc.2007.07.086

17. Navaneethan SD, Schold JD, Walther CP, et al. High-density lipoprotein cholesterol and causes of death in chronic kidney disease. $J$ Clin Lipidol. 2018;12(4):1061-1071.e7. doi:10.1016/j.jacl.2018. 03.085

18. Upmeier E, Lavonius S, Lehtonen A, et al. Serum lipids and their association with mortality in the elderly: a prospective cohort study. Aging Clin Exp Res. 2009;21(6):424-430. doi:10.1007/BF03327441

19. Oh IH, Hur JK, Ryoo JH, et al. Very high high-density lipoprotein cholesterol is associated with increased all-cause mortality in South Koreans. Atherosclerosis. 2019;283:43-51. doi:10.1016/j. atherosclerosis.2019.01.035

20. Hirata A, Okamura T, Sugiyama D, et al. The relationship between very high levels of serum high-density lipoprotein cholesterol and cause-specific mortality in a 20-year follow-up study of Japanese general population. J Atheroscler Thromb. 2016;23(7):800-809. doi: $10.5551 /$ jat. 33449

21. Li ZH, Lv YB, Zhong WF, et al. High-density lipoprotein cholesterol and all-cause and cause-specific mortality among the elderly. $J$ Clin Endocrinol Metab. 2019;104(8):3370-3378. doi:10.1210/jc.201802511

22. Mazidi M, Mikhailidis DP, Banach M. Associations between risk of overall mortality, cause-specific mortality and level of inflammatory factors with extremely low and high high-density lipoprotein cholesterol levels among American adults. Int J Cardiol. 2019;276:242247. doi:10.1016/j.ijcard.2018.11.095

23. Madsen CM, Varbo A, Nordestgaard BG. Extreme high high-density lipoprotein cholesterol is paradoxically associated with high mortality in men and women: two prospective cohort studies. Eur Heart J. 2017;38(32):2478-2486. doi:10.1093/eurheartj/ehx163

24. Ganjali S, Momtazi-Borojeni AA, Banach M, et al. HDL functionality in familial hypercholesterolemia: effects of treatment modalities and pharmacological interventions. Drug Discov Today. 2018;23 (1):171-180. doi:10.1016/j.drudis.2017.09.014

25. Ganjali S, Blesso CN, Banach M, et al. Effects of curcumin on HDL functionality. Pharmacol Res. 2017;119:208-218. doi:10.1016/j. phrs.2017.02.008 


\section{Publish your work in this journal}

Clinical Interventions in Aging is an international, peer-reviewed journal focusing on evidence-based reports on the value or lack thereof of treatments intended to prevent or delay the onset of maladaptive correlates of aging in human beings. This journal is indexed on PubMed Central, MedLine, CAS, Scopus and the Elsevier
Bibliographic databases. The manuscript management system is completely online and includes a very quick and fair peer-review system, which is all easy to use. Visit http://www.dovepress.com/ testimonials.php to read real quotes from published authors. 